\title{
Una experiencia en la formación de docentes a partir de la historia y la filosofía de la química
}

\author{
José Antonio Chamizo \\ Facultad de Quimica-Instituto de Investigaciones Filosóficas, Universidad Nacional Autónoma de México, \\ Ciudad de México, México \\ jchamizo@unam.mx \\ ORCID: https:// orcid.org/0000-0003-2719-5437
}

Juan Carlos García

School for the Future of Innovation in Society, Arizona State University, Tempe, Estados Unidos

j.carlos.garcia.c@gmail.com

ORCID: https:// orcid.org/0000-0002-6707-5555

[Recibido: 11 Febrero 2019. Revisado: 1 Junio 2019. Aceptado: 12 Julio 2019]

\begin{abstract}
Resumen: Se describe una experiencia de formación con docentes de bachillerato especializados en química. Para ello se parte de una posición filosófica centrada en las prácticas que considera, además de una discusión sobre textos específicos, llevar a cabo dos pequeñas investigaciones una de carácter histórico y otra de carácter filosófico. Ambas son presentadas y analizadas públicamente.
\end{abstract}

Palabras clave: Formación de docentes, Historia y Filosofía de la Química, Filosofía de la ciencia centrada en prácticas, diagramas heurísticos.

An experience in teacher training based on the history and philosophy of chemistry

Abstract: A training experience is described with high school teachers specialized in chemistry. From a philosophical position centered on the practices, it is considered, in addition to a discussion on specific texts, to carry out two small investigations, one of a historical nature and the other of a philosophical nature. Both are presented and analyzed at the end of the course with all participants.

Keywords: Teacher training, History and Philosophy of Chemistry, Philosophy of science centered on practices, heuristic diagrams

Para citar este artículo: Chamizo, J.A. y García-Cruz, J.C. (2020). Una experiencia en la formación de docentes a partir de la historia y filosofía de la química. Revista Eureka sobre Enseñanza y Divulgación de las Ciencias 17(1), 1601. doi: 10.25267/Rev_Eureka_ensen_divulg_cienc.2020.v17.i1.1601

\section{Introducción}

A pesar de lo mucho que ya se sabe, la práctica de la educación química en las escuelas no ha considerado de manera relevante los resultados de la investigación educativa. Por ejemplo, la manifiesta persistencia de conceptos alternativos y generalmente erróneos desde la propia disciplina (Kind 2004), la importancia de la explicitación y uso de los modelos y el modelaje (Chamizo 2010; Seok, 2011), el valor de la investigación experimental (Hodson 1994; Izquierdo 1999; Nakaleh 2002; Psillos 2002; Caamaño 2004; Nieto 2013) o la necesidad de incorporar y trabajar en un contexto determinado (Gilbert 2006; Brzezinski 2018) siguen prácticamente ausentes. Más aún, los cambios que han ocurrido en la mayoría de los libros de texto durante las últimas tres décadas, en general, no muestran un reconocimiento real del crecimiento en el conocimiento químico (una excepción a esta tendencia es, por ejemplo, el libro Cutting Edge Chemistry publicado por la Royal Society of Chemistry ihace casi dos décadas!) e ignoran aspectos sociales y filosóficos de su desarrollo (Moreno y Calvo 2019).

Reconociendo lo anterior, pero también identificando algunas de sus causas, se ha venido gestando un cambio curricular (Schulz 2014; Chamizo y Pérez 2017) en el que se está

\author{
Revista Eureka sobre Enseñanza y Divulgación de las Ciencias \\ Universidad de Cádiz. APAC-Eureka. ISSN: 1697-011X \\ http://dx.doi.org/10.25267/Rev_Eureka_ensen_divulg_cienc.2020.v17.i1.1601 \\ http:/ / reuredc.uca.es
}


desplazando el interés del aprendizaje de "ciencias a través de la educación" que ha llevado a aprender de memoria, una amplia "retórica de conclusiones" (Schwab 1962), por otro basado en las necesidades sociales de la educación, cercano a la alfabetización científica externalista (Gil y Vilches 2006; Acevedo 2008; Hodson 2014; Norris 2014). Este cambio curricular apunta a la "educación a través de las ciencias" como se ejemplifica en aprender a resolver problemas (Watts 1991; Izquierdo 2005; Holbrook y Rannikmae 2007; Chamizo 2013). Así, contrariamente a la idea de aprender conceptos específicos y aislados de la disciplina, por ejemplo en química la estructura atómica con todos los problemas reconocidos que ello conlleva (Chamizo y Garritz 2017), el objetivo fundamental es la educación integral del individuo. Así las ciencias no son más que uno de los vehículos para alcanzar lo que Claxton denominó hace ya muchos años "las capacidades de los jóvenes para vivir vidas interesantes, satisfactorias, dignas y agradables" es decir:

"En el mundo en el que van a vivir los jóvenes, nada podría tener más valor que la capacidad de construir nuestra propia vida a medida que la vivimos: encontrar nosotros mismos qué es lo que nos satisface, conocer nuestros propios valores y nuestra propia mente, enfrentarnos a la incertidumbre con coraje e ingenio, y valorar lo que nos dicen los demás con un escepticismo inteligente y sano.... La preocupación fundamental de una educación contemporánea útil debe centrase en la capacidad de las personas de aprender bien. Cualquier otra prioridad, por muy apreciada que sea, que socave el compromiso de fomentar la habilidad para el manejo del cambio o nuestro éxito en hacerlo, deberá ser relegada o suspendida." (Claxton 1994, p. 159).

Relacionado con esto se encuentra la extendida creencia de que los contenidos de los libros de texto de ciencias son, de hecho, ciencia. Lo anterior no es necesariamente cierto. Muchos de los materiales escritos empleados en la educación científica alrededor del mundo son descripciones simplificadas y descontextualizadas de exploraciones científicas pasadas, es decir muchos libros de texto son, sin decirlo, libros de historia de las ciencias. Por eso, entre otras razones, hay que abordar el tema de la historia y la filosofía de las ciencias en la formación de docentes y aquí lo hacemos a partir de las prácticas (Turner 1994). Las prácticas consideran un saber tácito propio de las diferentes actividades de investigación que poco a poco han ido reconociéndose como fundamentales en la filosofía de las ciencias (Polanyi 1966; Martínez 2015).

\section{Sobre la historia y filosofía de las ciencias}

Seguramente fue T. Kuhn, más que ningún otro filósofo, el que insistió en la importancia de la educación en la conformación de lo que él llamó 'ciencia normal': Sin embargo, puesto que los libros de texto son vebiculos pedagógicos para la perpetuación de la ciencia normal, siempre que cambien el lenguaje, la estructura de los problemas, o las normas de la ciencia normal, tienen integramente, o en parte, que volver a escribirse. (Kuhn 1971, p. 214).

Así la química de los libros de texto en prácticamente todo el mundo responde a lo que ha sido identificado como una posición dominante (Van Brakel 2000) y cuya característica sustantiva-filosófica-pedagógica es: Todo el currículum de química actual tiene una estructura sustantiva dominante basada en la teoría corpuscular, la cual es rígidamente combinada con una estructura filosófica específica, el positivismo, y una estructura pedagógica específica, la iniciación y preparación de los futuros químicos profesionales.

Reconocer esta estrecha posición dominante coincide con lo dicho por el mismo Kuhn:

“...los libros de texto no abordan los problemas que los científicos profesionales enfrentan o la variedad de técnicas que la experiencia les ha mostrado son capaces de 
utilizar para resolverlos. En su lugar, los libros de texto, exhiben una colección de problemas-solución que los científicos profesionales han aceptado como paradigmáticos, pidiéndosele a los alumnos que, ya sea con lápiz y papel o en el laboratorio, los resuelvan utilizando los métodos y/o sustancias que han mostrado con anterioridad en sus páginas." (Kuhn, 1963, pp. 350-351).

La ciencia normal privilegia el trabajo técnicamente preciso y lógicamente riguroso y es alrededor del cual, ignorándolo, se forman los docentes y los alumnos en la mejor tradición del positivismo lógico (Van Aalsvoort 2004). Así se puede interpretar el currículo de química como educación científica normal con las siguientes características:

- La educación científica normal prepara a los estudiantes para hacer ciencia normal.

- La educación científica normal es la forma dominante y normal en la que se enseñan las ciencias en prácticamente todos los niveles, lo cual la hace paradigmática.

- La educación científica normal contiene, de manera implícita, normas respecto a la ciencia, la filosofía y la pedagogía.

Una característica importante del currículo actual, que por su implantación en prácticamente todo el mundo podríamos identificar como paradigmático, es la rígida relación existente entre las temáticas, las posturas filosóficas inherentes a la química y la pedagogía. Lo anterior se manifiesta en la principal conclusión de la investigación de van Brakel (2000, p. 123): " $L a$ educación química normal está aislada del sentido común, de la vida cotidiana, de la sociedad, de la historia y filosofía de la ciencia, de la tecnología, de la física escolar y de la investigación química actual". Todo lo anterior coincide con la insistente petición de incorporar la historia y la filosofía de las ciencias en los currículos escolares y en los de formación de docentes (Hodson 2008; Höttecke 2011; Mathews 2014) y en particular en los de química (Wandersee 2002; Erduran 2007, 2013; Gallego 2009; Tolvanen 2014; Justi 2016; Moreno y Calvo 2019; Gois en prensa). En este texto pretendemos indicar una manera de cómo hacerlo.

\section{Sobre las prácticas académicas}

En principio, debemos profundizar en la concepción de prácticas académicas. Uno de los críticos de la concepción positivista del conocimiento fue L. Wittgenstein. En sus Investigaciones filosóficas (1988) y luego en Sobre la certeza (1991), Wittgenstein hizo hincapié en la importancia que tiene tomar en consideración las prácticas en distintas áreas de la filosofía y, en particular, para elucidar el problema en torno a su relación con el conocimiento. Estas críticas suscitaron varias preguntas básicas para la teoría del conocimiento, porque si aceptamos la idea de que las prácticas son relevantes para la reflexión epistemológica, entonces cabe preguntarse: ¿qué papel desempeñan las prácticas en conexión con la aceptabilidad epistémica? o particularmente para este trabajo respecto a la formación docente ¿cuál es la importancia de adquirir ciertos conocimientos para una determinada práctica?

En su libro The Social Theory of Practices (1994), Turner indica que una explicación en términos de prácticas requiere que se justifique la inferencia de comportamiento coordinado manifestada en regularidades observables - a su supuesto origen en presupuestos o prácticas compartidas. Para Turner, de acuerdo con Olivé (2008) las prácticas sólo podrían entenderse como "ocultas" y, por lo tanto, resulta imposible dar cuenta de cómo esas prácticas, qua prácticas, se transmiten de individuo a individuo; en lugar de prácticas, debería entonces hablarse de algún tipo de presuposición cognitiva, que es lo que sería compartido, y ésta sería la causa (y punto de acceso al entendimiento) de las manifestaciones compartidas que observamos. Otra crítica de Turner es que el término práctica se utiliza para referirse a muchas cosas: "tradiciones", "conocimiento tácito", "paradigma", "presuposición", "competencias no 
articulables explícitamente", etc. No obstante, Olivé plantea un contraargumento genuino con la idea de cierta clasificación de prácticas, es decir una práctica depende, siempre, de cierta clasificación de prácticas que da por sentado conocer otras prácticas y, más en general, una serie de presupuestos culturales que permiten distinguir una práctica de otra (Olivé 2008). Por ejemplo en la enseñanza de la química las prácticas experimentales son imprescindibles.

Por tanto, aquí entendemos por práctica al conjunto de acciones coordinadas por ciertos "nexos" tales como a) comprensión de qué hacer y qué decir; b) conjuntos de reglas, principios, preceptos e instrucciones; c) estructuras que incluyen fines, proyectos, tareas, propósitos, creencias, emociones y estados de ánimo (Schatzki 1996). Más específicamente, nos situaremos en una práctica científica que incluyen: (1) formas de comprender tipos de acciones; (2) reglas explícitas, principios, preceptos e instrucciones; y (3) estructuras "teleoafectivas", que contienen jerarquías de fines, tareas, propósitos, proyectos, creencias, emociones y estados de ánimo. Al conjunto de formas de comprensión, reglas y estructura teleoafectiva, Schatzki (1996) lo nombra la "organización” de la práctica.

En suma, podemos comprender la práctica académica vinculada a sistemas más amplios en cuatro grandes contextos de la práctica científica: a) académica-educativa, b) investigación, c) aplicación y d) evaluación, dentro de cada uno de los cuales y en la interacción entre ellos se producen distintas actividades científicas (Olivé 2008). Así pues, la práctica académica se constituye como un complejo sistema de actividades (acciones, reacciones, interacciones, hábitos, operaciones, etc.) procedentes de una prolongada tradición cultural: la cultura científica, orientada al logro de conocimientos.

\section{Metodología}

En este trabajo se describen las prácticas académicas desarrolladas en la asignatura obligatoria "Fundamentos teóricos metodológicos: Filosofía e Historia de la química" impartida en el programa de Maestría en Docencia para la Educación Media Superior (MADEMS) con sede en la Facultad de Química de la Universidad Nacional Autónoma de México (UNAM). La matrícula de estudiantes proviene de áreas profesionales de la química en su mayoría, a excepción de un par de semestres donde se ofertó como asignatura opcional en la Maestría en Filosofía de la Ciencia impartida en el Instituto de Investigaciones Filosóficas de la UNAM. A lo largo de estos 8 años la han cursado ochenta estudiantes, la mayoría de ellos docentes en activo de bachillerato. Aquí los reconoceremos como alumnos-docentes.

\section{Prácticas académicas en la asignatura Historia y Filosofía de la Química}

La asignatura inició con el objetivo general de examinar y discutir algunos temas centrales de los estudios filosóficos, históricos y sociales sobre la ciencia y la tecnología. Sin embargo, durante el desarrollo de esta asignatura, y ante el manifiesto y repetido desconocimiento de los docentes sobre estos temas, se han ido modificando sus objetivos hasta examinar principalmente el papel de la historia y la filosofía de la química como requisito indispensable en la formación de los docentes de esta asignatura. Así consideramos necesario, además de la lectura y discusión de textos específicos, particularmente aquellos relacionados con la historia de la química (Chamizo 2018; Anexos), que los docentes-alumnos demuestren la apropiación de la temática de la asignatura a través de dos actividades, o de acuerdo con lo aquí discutido dos prácticas diferentes.

La primera, una investigación histórica de algún tema relacionado con la química y que se concreta a través de la elaboración de un diagrama heurístico (Chamizo 2012, 2017) cuya utilidad en procesos de formación de docentes ha sido documentada (Chamizo y GarcíaFranco 2013). La segunda actividad, consiste en la investigación y elaboración de un ensayo 
crítico filosófico (Nubiola 2009; Pryor 2012) que exponga e identifique un práctica química y su relación con un problema filosófico. Ambas actividades inician con la identificación del tema a investigar y que cada alumno-docente elige. Posteriormente 6 semanas después, se tiene una discusión pública y compartida sobre sus avances en ambos procesos de investigación en los que reciben una importante retroalimentación, tanto por los responsables del curso como por los propios compañeros, y los resultados de la misma son presentados al final del curso también en una sesión pública. A continuación se presentan las particularidades de ambas actividades así como ejemplos de su uso a lo largo del curso.

\section{Investigación Histórica. Diagrama heurístico}

Los diagramas heurísticos son organizadores gráficos (Trowbridge, 1998) derivados de los propuestos originalmente por Novak y Gowin (1984) en los que a partir de unos hechos significativos se elabora una pregunta abierta y se diferencia explícitamente, para resolverla, entre dos dominios el conceptual y el metodológico. Con el apoyo de una rejilla de autoevaluación que permite identificar la meta-cognición, y que es una de las estrategias más importantes para mejorar el logro educativo (Hattie, 2009), los Diagramas Heurísticos se han empleado para concretar en una sola cuartilla el inicio, proceso y resultado de una investigación. Ésta puede ser experimental, educativa o histórica desde el nivel de bachillerato hasta el de posgrado (Chamizo 2013). Permiten evaluar habilidades de pensamiento científico (Chamizo 2017) a través de lo que se ha denominado "cartografía de la cognición" (Wandersee, 1990). En el aula se construye así una comunidad de prácticas una vez que se convoca a los alumnos-docentes a establecer "un compromiso mutuo, una empresa conjunta y un repertorio compartido" (Capobianco and Feldman, 2006, p. 502) donde cada alumnodocente funciona como compañero crítico de los demás, una vez que todos ellos comparten continuamente los avances obtenidos en su investigación. En los Anexos se presentan dos diagramas heurísticos que concretan investigaciones realizadas por alumnos-docentes de este curso y que ejemplifican claramente el trabajo que realizaron. Los problemas más frecuentes en su elaboración son:

- Reconocimiento de entre todos los hechos históricos relacionados con su tema, los tres o cuatro más significativos para elaborar la pregunta.

- Construir una pregunta abierta, es decir cuya respuesta no este en un único documento y que requiera reunir conceptos y metodología.

- Identificar el modelo adecuado para responder desde él mismo la pregunta (Chamizo y García-Franco 2013). En este punto los alumnos-docentes aprenden a que hay diversidad de modelos, históricos, económicos, científicos, etc. y que es a partir del modelo que elijan la respuesta que pueden dar.

- Para una de las alumnas-docentes representó una actividad muy intensa. En sus propias palabras: "Bueno sobra decir que el ejercicio de construir un diagrama heurístico, implicó una tarea titánica de pensamiento y abstracción, que hasta hoy, recuerdo con cariño. Pues al final de la aventura, aprendí y digerí a fondo".

Hay que insistir en que los diagramas heurísticos informan una investigación que es producto de una comunidad de prácticas, de un proceso iterativo compartido entre los alumnosdocentes, los responsables del curso y ellos mismos.

\section{Investigación Filosófica. Ensayo crítico filosófico}

Comprendemos por ensayo crítico el arte de juzgar en el contexto de verdades provisionales en el tiempo. Es decir, la reflexión individual frente a la argumentación de la otredad. Es el sustento de una tesis personal que analiza textos y documenta evidencias que críticamente 
sostienen el refutar y presentar los nuevos argumentos (Ochoa 2007). El sustento no es la estructura del ensayo, sino las piezas de la armazón argumentativa que en la reflexión generan las nuevas ideas que ayudan a comprender las actividades y prácticas que desarrolla la actividad tecnoquímica. Así pues, el ensayo implica, necesariamente, la argumentación e incorporar la argumentación en los entornos de aprendizaje de la ciencia mejora la comprensión conceptual y epistemológica (Jonassen y Bosung 2010; Jaramillo y Mendoza 2004). En esta vertiente, la investigación inicia con el planteamiento de una pregunta de investigación y la tesis a desarrollar. A continuación podemos revisar el siguiente ejemplo elaborado por un alumno relacionado con una postura realista sobre ciertas nociones usadas en química (por ejemplo la existencia de los átomos). La pregunta de investigación construida por el alumno fue: ¿Qué consecuencias epistémicas conlleva asumir conceptos como relación causa-efecto o propiedades de la materia que no tienen realidad ontológica o que son meras ficciones? La importancia de plantear una pregunta viable y fecunda de investigación nos lleva a preguntarnos qué elementos del curso, lecturas y referencias necesitamos para argumentar la posible respuesta. En este sentido, la metodología a desarrollar implica dos vertientes una es la parte objetiva (o científica) y la subjetiva (o literaria) (Paul y Elder 2003; Alzate 2009). Así, el ensayo siempre cuenta con la parte científica porque relaciona los hechos y la parte subjetiva o literaria por contar con el juicio crítico del ensayista. En este sentido, la primera parte debe apoyarse en al menos ocho referencias revisadas a lo largo del curso, eso con el objetivo de reconocer la apropiación del alumno de la lectura, de las referencias y cada una de la sesiones de la discusión (Apéndice).

En consecuencia el ensayo es, entonces, la unión casada de dos mundos: el de la ciencia y el de la originalidad del ensayista para generar respuestas que nos permitan comprender mejor el campo histórico y filosófico de la actividad química. Por un lado, el alumno profundiza en las referencias revisadas del curso y por otro lado, propone creativamente otras referencias que lo apoyan en la construcción de la respuesta planteada. Con ello se supone una posición con respecto al tema, lo que motiva a la reflexión y la emisión de juicios que comprometen al autor en la búsqueda de estas respuestas, lo que impulsan a formular esas preguntas que lo llevan a relacionar sus ideas con las que están disponibles en las fuentes, encontrar vínculo entre lo que ya sabe y lo que necesita saber, a crear nuevas relaciones entre los datos disponibles, su contexto y propósito (Cañón 2002).

En esta vertiente el ensayo cuenta con tres secciones: el planteamiento, el desarrollo y las conclusiones. En el planteamiento, se les solicita a los alumnos la exposición del problema y la tesis a defender; ello les permite delimitar alcances y limitaciones, mostrar su pertinencia, identificar las fuentes de su problema de investigación en el que exista un conflicto y el autor se proponga mostrar su opinión acerca del tema, aportando evidencias y argumentos para sustentarla. En este sentido, la problemática sobre la cual inician su argumentación parte de la temática general sobre la filosofía de la química. Para continuar con nuestro ejemplo, veamos el planteamiento elaborado:

"Diversos estudios sociales sobre las ciencias y sus prácticas, llevados a cabo principalmente en el siglo XX, pusieron en tela de juicio algunas ideas arraigadas dentro de estas actividades, como la neutralidad o la objetividad de los datos observados. Una tesis negativa consiste en asumir que ciertos conceptos como la relación causa-efecto o las propiedades de la materia, no tienen realidad ontológica y son meras ficciones. En este breve ensayo se argumenta a favor de una postura realista de estas nociones enfatizando su uso en la química".

En el ejemplo podemos observar que la delimitación versa en el planteamiento de los argumentos para resolver la pregunta, basada en los textos revisados en el curso. En esta 
vertiente en el desarrollo, la segunda parte del trabajo, se presenta la defensa de la tesis a través del análisis de los argumentos que versan entorno a las posturas que tiende a defender la tesis. En este punto ha sido sumamente importante retomar por lo menos ocho referencias revisadas en clase; debido a la importancia de la discusión y el seguimiento de un problema delimitado y discutido a lo largo del semestre.

Finalmente, la conclusión, se estructura a través de regresar a la primera parte, el planteamiento del problema, con la finalidad de resaltar la importancia de los hechos que validaron la hipótesis, para finalmente atar cabos y dar una respuesta a las interrogantes planteadas. En este sentido, podemos observar las conclusiones del trabajo que nos ha servido como ejemplo:

"Una postura intermedia sobre la realidad de ciertas nociones que no pueden considerarse como características de los fenómenos naturales como el de causalidad, puede conciliar dos puntos de vista incompatibles, aquel que considera meras ficciones algunos conceptos e ideas empleados en las prácticas con otro que asevere la realidad ontológica de éstos. Estas nociones pueden considerarse derivadas del contexto en el que se emplean, y se puede asumir su realidad del mismo modo que se asume la realidad de los puentes de hidrógeno, es decir, se asumen su realidad en virtud de que se está investigando o experimentando en un dominio restringido y en un contexto particular bajo determinados intereses. Sin embargo, también se debe asumir que la química considerada así es una química que se asemeja más a la que se practicaba y concebía en tiempos de Lavoiser que la de los químicos actuales y que su función sólo obedece a ciertos propósitos prácticos como la didáctica o la heurística."

Como podemos observar el trabajo es el resultado de una reflexión y discusión de una serie de lecturas que aportan al estudiante la posibilidad de comprender un problema a través de los marcos conceptuales revisados en el curso.

Incorporar referencias actualizadas nos ha permitido responder a la diversidad de intereses de los alumnos en tópicos referentes a la filosofía e historia de la química, campo que indudablemente se encuentra en constante construcción y que debemos incentivar con trabajos que profundicen en alguna de las temáticas. En la Tabla 1 se indican algunos de los títulos y resúmenes de los trabajos realizados por los alumnos-docentes a lo largo de los años en los que se ha llevado a cabo esta experiencia, en los que se podrá reconocer la importancia que le dan a reflexionar sobre su propia práctica docente (Rivard 2000). 
Tabla 1. Nombres y resúmenes de ensayos escritos por los alumnos-docentes.

\begin{tabular}{|c|c|}
\hline Título del ensayo filosófico & Resumen \\
\hline $\begin{array}{l}\text { El dogmatismo inherente a la ciencia como } \\
\text { obstáculo epistemológico }\end{array}$ & $\begin{array}{l}\text { Este ensayo expone de manera explícita las limitaciones, } \\
\text { aporías, axiomas, que por definición se tornan en } \\
\text { dogmas inherentes al conocimiento científico, así como } \\
\text { hacer explícitas las dependencias directas e indirectas } \\
\text { con respecto a otras ramas del conocimiento, como } \\
\text { parte fundamental de la educación en química, se corre } \\
\text { el riesgo de generar una cerrazón conservadora, guiada } \\
\text { por la necesidad de seguridad, en la labor de adquisición } \\
\text { del conocimiento, lo cual puede ser entendido como un } \\
\text { obstáculo epistemológico, en el sentido planteado por } \\
\text { Gaston Bachelard. }\end{array}$ \\
\hline $\begin{array}{l}\text { El papel de la filosofía de la química en la } \\
\text { enseñanza de la química }\end{array}$ & $\begin{array}{l}\text { Este ensayo elaborara una prueba que permita } \\
\text { identificar aquellas concepciones que pueden } \\
\text { representar un obstáculo para la comprensión de las } \\
\text { teorías científicas en la química. }\end{array}$ \\
\hline $\begin{array}{l}\text { La concepción del experimento y su influencia en } \\
\text { la enseñanza de la química en el bachillerato }\end{array}$ & $\begin{array}{l}\text { Este ensayo reivindica, desde la epistemología, el lugar } \\
\text { que le corresponde al experimento como una actividad } \\
\text { útil en el desarrollo de los aspectos científicos de la } \\
\text { cultura que se consideran necesarios para la formación } \\
\text { de los estudiantes del bachillerato, partiendo del hecho } \\
\text { de que en la actualidad, el modelo tradicional de } \\
\text { enseñanza relega al experimento a una operación que } \\
\text { carece de significado, pues en el aula difícilmente se } \\
\text { discuten las características de esta actividad. }\end{array}$ \\
\hline $\begin{array}{l}\text { Problemas de la transformación del mundo. Las } \\
\text { costumbres y tradiciones originarias de la mixteca } \\
\text { de Oaxaca en el proceso químico }\end{array}$ & $\begin{array}{l}\text { Este ensayo analiza la viabilidad de que las costumbres y } \\
\text { tradiciones originarias de México puedan ser retomadas } \\
\text { por las comunidades científicas, específicamente por las } \\
\text { prácticas químicas para incentivar los procesos de } \\
\text { investigación. }\end{array}$ \\
\hline $\begin{array}{l}\text { La ciencia escolar, el gran reto de su enseñanza en } \\
\text { la educación a nivel Medio Superior; caso particular } \\
\text { de la química }\end{array}$ & $\begin{array}{l}\text { Este ensayo aborda la enseñanza de la ciencia (química) } \\
\text { en México en el Nivel Medio Superior. Para intervenir, } \\
\text { se plantea la hipótesis de que si a la ciencia escolar se le } \\
\text { incluye una imagen histórica-filosófica el alumno } \\
\text { involucrará mayores competencias con lo cual será más } \\
\text { crítico y reflexivo y con ello mejorar la percepción de la } \\
\text { enseñanza de las ciencias en el Nivel Medio Superior. }\end{array}$ \\
\hline $\begin{array}{l}\text { Ensayo de ¿Son éticamente neutrales la ciencia y la } \\
\text { tecnología? }\end{array}$ & $\begin{array}{l}\text { Este ensayo parte de la pregunta ¿Es posible la } \\
\text { neutralidad ética de la ciencia y la tecnología? Para lo } \\
\text { cual se realizará un análisis de que aspectos las } \\
\text { constituyen y las desarrollan las prácticas. }\end{array}$ \\
\hline $\begin{array}{l}\text { De la metáfora al modelo "modelos químicos y su } \\
\text { interpretación" }\end{array}$ & $\begin{array}{l}\text { Este ensayo revisa los enunciados y modelos } \\
\text { metafóricos del enlace químico, uno de los temas más } \\
\text { abordados en la disciplina de química, contextualizados } \\
\text { desde una visión del realismo científico. También se } \\
\text { examina el sentido literal que los estudiantes de química } \\
\text { le dan a las metáforas científicas en relación con la } \\
\text { realidad, convirtiéndose este en un obstáculo analógico } \\
\text { heredado por una enseñanza tradicionalista que ha } \\
\text { convertido a los enunciados teóricos en modelos } \\
\text { metafóricos. }\end{array}$ \\
\hline $\begin{array}{l}\text { La enseñanza de la nomenclatura en química } \\
\text { orgánica desde un enfoque realista y a partir de la } \\
\text { propuesta de Lakatos }\end{array}$ & $\begin{array}{l}\text { Este ensayo propone que la comprensión de la } \\
\text { nomenclatura en química orgánica por parte de los } \\
\text { alumnos de nivel medio superior y superior puede verse } \\
\text { superados si se considera para su enseñanza un análisis } \\
\text { histórico y epistemológico, en este caso utilizando la } \\
\text { propuesta de Lakatos sobre los programas de } \\
\text { investigación. }\end{array}$ \\
\hline
\end{tabular}


Tabla 1. Continuación

\begin{tabular}{|c|c|}
\hline Título del ensayo filosófico & Resumen \\
\hline $\begin{array}{l}\text { El químico su estilo de aprendizaje abordando la } \\
\text { filosofía de la ciencia y de la química. }\end{array}$ & $\begin{array}{l}\text { En el presente ensayo se hace una descripción de lo que } \\
\text { son los químicos, el estilo de aprendizaje que prefieren y } \\
\text { que elementos se deben tomar en cuenta para abordar la } \\
\text { filosofía de la ciencia y de la química y se concluye con } \\
\text { una postura filosófica. }\end{array}$ \\
\hline ¿Existen los iones? & $\begin{array}{l}\text { El presente trabajo toma como punto de partida, cómo el } \\
\text { que hacer de los químicos no se ve influenciados por la } \\
\text { pregunta existencialista sobre las entidades que forman su } \\
\text { ciencia, las cuales se aceptan como reales, y el no creer en } \\
\text { su existencia es prácticamente ir en contra de } \\
\text { la Química. Así pues se abordan las posturas realistas y } \\
\text { anti realistas sobre estas entidades, y se defiende la } \\
\text { postura realista sobre la anti realista, argumentando las } \\
\text { diferencias, similitudes y debilidades de la posición anti } \\
\text { realista en este debate. }\end{array}$ \\
\hline $\begin{array}{l}\text { La enseñanza aprendizaje de la tecnoquímica en } \\
\text { los cursos de bachillerato }\end{array}$ & $\begin{array}{l}\text { Este ensayo analiza la formación, la escuela y los docentes } \\
\text { juegan un papel muy importante en la impartición de } \\
\text { cursos de ciencias, humanidades y artes; particularmente } \\
\text { en la formación científica, los cursos de ciencias naturales } \\
\text { juegan un papel fundamental, como es el caso de la } \\
\text { química. ciencia, tecnología y sociedad. }\end{array}$ \\
\hline ¿Química o Tecnociencia? & $\begin{array}{l}\text { Este ensayo gira alrededor de la tesis de que los químicos } \\
\text { en general no están acostumbrados al término } \\
\text { tecnociencia, y que pocas veces relacionan su disciplina } \\
\text { con este término; para ellos la Química es una ciencia en } \\
\text { el sentido descrito por la postura del positivismo lógico. } \\
\text { Y en el contexto actual, se debe tratar de ver a la Química } \\
\text { desde una perspectiva diferente a la heredada de esta } \\
\text { postura filosófica, pues esta no describe íntegramente la } \\
\text { esencia de la Química. }\end{array}$ \\
\hline
\end{tabular}

\section{Consideraciones finales}

Caracterizar y describir las prácticas de una asignatura es complejo, pero todavía más si observamos que en pocas universidades y posgrados han incentivado la enseñanza de la química desde una óptica filosófica e histórica. No obstante, estamos convencidos que este ejemplo muestra la importancia de incluir la historia y la filosofía de las ciencias en su propia enseñanza ya no se pone en duda como quedo establecido hace más de sesenta años (Conant 1957). Por ejemplo para mostrar la experiencia del 'International History and Philosophy of Science Teaching Group', desde 1989 se han llevado a cabo 15 conferencias internacionales (además de muchas regionales), a partir de 1992 se publica una revista específicamente dedicada al tema (Science \& Education) y hace cinco años un Handbook (Matthews 2014) con la autoria de 125 profesores de 30 países. Sin embargo aún hay resistencia de muchos docentes de permitirse entender la enseñanza desde otro lugar diferente al que ellos mismos conocen (Bourdieu y Passeron, 1995). Por ello un curso de humanidades en una clase con alumnosdocentes de ciencias necesita, para aspirar a tener un mínimo éxito, el que los participantes hagan suya alguna de las prácticas de las disciplinas involucradas. Evidentemente esa práctica es superficial pero sin duda indicativa del saber hacer, en este caso de la historia y la filosofía. En nuestro caso los alumnos-docentes se cuestionaron y profundizaron sobre sus propios conocimientos de química lo cual redundará en sus futuros alumnos de bachillerato. Pero más aún, a través de las dos investigaciones realizadas y su presentación compartida reconocieron otras maneras de aprehender, tomar, o agarrar, el saber del mundo. Lo anterior, más allá del contexto de justificación donde la ciencia puede caracterizarse sólo en términos de valores 
epistémicos relativos a criterios para la elección de teorías. Es útil recordar la distinción trazada por H. Reichenbach en Experience and Prediction de 1938, donde en el contexto de descubrimiento se aluden a cuestiones relacionadas con la forma en que generamos nuevas ideas o hipótesis en ciencia; mientras que el contexto de justificación tiene que ver con el tipo de criterios que dichas hipótesis deben satisfacer para ser aceptadas en el corpus científico. Así, en el caso del contexto de descubrimiento se trata de exhibir los procesos psicológicos y sociales que tienen lugar durante el proceso real en que los científicos generan nuevas ideas o hipótesis. Por lo tanto, la práctica académica se constituye como un complejo sistema de actividades (acciones, reacciones, interacciones, hábitos, operaciones, etc.) procedentes de una prolongada tradición científica, cultural, social, educativa y química que profundizamos cada semestre en la asignatura historia y filosofía de la química impartida en la Maestría en Docencia Media Superior (MADEMS) en la Facultad de Química de la UNAM. Una de nuestras alumnas-docentes lo dice así:

"El curso de Historia y Filosofía de la Química me ha permitido acercarme a la química desde una mirada más cercana a su naturaleza, concibiendo así los conceptos y teorías propios de la disciplina como modelos creativos que han evolucionado impactados por las condiciones sociales, económicas y políticas en las que fueron desarrollados, de la misma manera en que se ven impactadas todas las empresas humanas. Estoy convencida de que en la medida en que los docentes nos interesemos en cómo se construye el conocimiento y cómo opera y evoluciona la ciencia, podremos desarrollar mejores estrategias didácticas para acercar el conocimiento a los estudiantes. Pero sobre todo, de que la manera en que percibimos la ciencia los profesores, permea en la actitud que desarrollen los estudiantes ante ella. Esto he podido aplicarlo de manera explícita en dos cursos que imparto actualmente en la Facultad de Química: Ciencia y Sociedad y Comunicación Científica. Contenidos que hoy en día, considero esenciales en la formación de futuros profesionales de la química."

\section{Agradecimientos}

Al Consejo Nacional de Ciencia y Tecnología (CONACyT) por el financiamiento otorgado a través del Programa EPE 2018, CVU: 173047.

\section{Referencias bibliográficas}

Acevedo J.A. (2008) El estado actual de la naturaleza de la ciencia en la didáctica de la ciencia, Revista Eureka de Enseñanza y Divulgación de las Ciencias, 5, 134-169.

Alzate Yepes T. (2009) Hay que enseñar a hacer ensayos. Revista Iberoamericana de Educación, 6(48), 1-5.

Bourdieu P., Passeron J.C. (1995) La reproducción. Elementos para una teoría del sistema de enseñanza. México: Fontamara.

Brzezinski M.E., Celestino C. (eds.) (2018) Teaching Science with Context. Historical Philosophical and Sociological Approaches. Dordrecht: Springer.

Caamaño A. (2004) Experiencias, experimentos ilustrativos, ejercicios prácticos e investigaciones. ¿Una clasificación útil para los trabajos prácticos? Alambique, 39, 8-19.

Cañón Vega N. (2002) Guía para la elaboración y presentación de trabajos escritos. Bogotá: Universidad de La Salle.

Chamizo J.A. (2010) Una tipología de los modelos para la enseñanza de las ciencias. Eureka, Revista de Enseñanza y Divulgación de las Ciencias. 7, 26-41. 
Chamizo J.A. (2012) Heuristic diagrams as a tool to teach history of science, Science \& Education. 21, 745-762.

Chamizo J.A. (2013) De la paradoja a la metáfora. La enseñanza de la química a través de sus modelos. México: UNAM-Siglo XXI.

Chamizo J.A. (2017) Habilidades de pensamiento cientifico. Los diagramas heurísticos. México: FQUNAM.

http://www.joseantoniochamizo.com/pdf/educacion/libros/014_Habilidades_pensa miento_cientifico.pdf

Chamizo J.A. (2018) Quimica General. Una aproximación histórica. México: FQ-UNAM. En línea: http://www.joseantoniochamizo.com/pdf/quimica/libros/002_Quimica_general.pdf

Chamizo J.A., García-Franco, A. (2010) Modelos y modelaje en la enseñanza de las ciencias. México: FQ-UNAM. En línea: http://www.joseantoniochamizo.com/pdf/educacion/libros/011_Modelos_modelaje _ensenanza_ciencias_naturales.pdf

Chamizo J.A., García-Franco, A. (2013) Heuristic diagrams as a tool to formatively assess teachers' research. Teachers and Teaching: Theory and Practice, 19, 135-149.

Chamizo J.A., Garritz A. (2014) Historical teaching of molecular and atomic structure en Matthews M. (ed) International Handbook of research in History Pbilosophy and Science Teaching. Dordrecht: Springer.

Chamizo J.A., Pérez Y. (2017) Sobre la enseñanza de las ciencias naturales. Revista Iberoamericana de Educación. 74, 23-40.

Claxton G. (1994) Educar mentes curiosas. El reto de la ciencia en la escuela. Madrid: Visor.

Conant J.B. (ed.) (1957) Harvard Case Histories in Experimental Science. 2 vols. Cambridge: Harvard University Press.

Erduran S. (2013) Philosophy, Chemistry and Education: An Introduction. Science \& Education, 7, 1559-1562.

Erduran S., Arduriz A. \& Naaman M, (2007) Developing epistemologically empowered teachers examining the role of philosophy of chemistry in teacher education. Science \& Education, 16, 975-989.

Gallego A.P., Gallego R., Pérez R. (2009) El Contexto Histórico Didáctico de la Institucionalización de la Química como Ciencia. Revista Eureka sobre Enseñanza y Divulgación de las Ciencias, 6, 247-263.

Gil D., Vilches A. (2006) Educación ciudadana y alfabetización científica. Mitos y realidades. Revista Iberoamericana de Educación, 42, 31-53.

Gois J., Pinto M.A. (ed). Filosofía da Química no Brasil. Universidade Regional do Nooeste do Estado do Rio Grande do Sul, en prensa.

Gilbert J. K. (2006) On the Nature of 'Context' in Chemical Education". International Journal of Science Education, 28, 957-976.

Hattie J. (2009) Visible Learning. A Synthesis of Over 800 Meta-Analyses Relating to Achivement, London: Routledge. 
Hodson D. (2014) Nature of Science in the Science Curriculum: Origin, Development, Implications and Shifting Emphases, en M. Matthews (ed) International Handbook of Research in History, Philosophy and Science Teaching. Dordrecht: Springer.

Hodson D. (2008) Towards Scientific Literacy: A Teachers' Guide to the History, Philosophy and Sociology of Science. Rotterdam: Sense Publishers.

Hodson D. (1994) Hacia un enfoque más crítico del trabajo del laboratorio. Enseñanza de las Ciencias, 12, 299-313.

Holbrook J., Rannikmae R. (2007) The nature of science education for enhancing scientific literacy. International Journal of Science Education, 29, 1347-1362.

Höttecke D., Silva C.C. (2011) Why implementing history and philosophy in school science education is a challenge: An analysis of obstacles. Science \& Education, 20, 293-316.

Izquierdo M. (2005) Resoldre problems per aprendre, EINES d' Innovació Docent en Educació Superior. Barcelona: Universitat Autònoma de Barcelona.

Izquierdo M., Sanmartí N., Espinet M. (1999) Fundamentación y diseño de las prácticas escolares de ciencias experimentales, Enseñanza de las Ciencias. 17, 45-59.

Jaramillo S., Mendoza V. (2004) Guía para la elaboración de ensayos de investigación. Razón y palabra, 41.

Jonassen D.H., Bosung K. (2010) Arguing to learn and learning to argue: design justifications and guidelines. Education Tech Research Dev 58, 439-457.

Justi R., Cardoso P.C. (2016) Discussion of the Controversy Concerning a Historical Event Among Pre-service Teachers. Science \& Education, 25, 795-822.

Kind V. (2004) Más allá de las apariencias. Ideas previas de los estudiantes sobre conceptos básicos de química. México: UNAM-Santillana.

Kuhn T. (1971) La Estructura de las Revoluciones Científicas. México: Fondo de Cultura Económica.

Kuhn T. (1963) The Function of Dogma in Scientific Research, en A.C. Crombie (ed) Scientific Change. London: Heineman.

Martínez S., Huang X. (2015) Hacia una filosofía de la ciencia centrada en prácticas. México: Bonilla Artigas-Instituto de Investigaciones Filosóficas UNAM.

Matthews M. (ed) (2014) International Handbook of Research in History, Philosophy and Science Teaching. Dordrecht: Springer.

Moreno L., Calvo M.A. (2019) ¿Cómo presentan la historia de la química los libros de texto de Educación Secundaria? Un análisis desde la didáctica y los estudios históricos de la ciencia. Revista Eureka de Enseñanza y Divulgación de las Ciencias 16, 1101.

Nakhleh M.B., Polles J., Malina E. (2002) Learning chemistry in a laboratory environment, en J. Gilbert (ed.) Chemical education: Towards Research-based Practice. Dordrecht: Kluwer.

Nieto E. y Chamizo J.A. (coordinadores) (2013) La Enseñanza Experimental de la Química. Las experiencias de la UNAM. México: Facultad de Química-UNAM.

Norris S. P., Philips L.M. and Burns D. (2014) Conceptions of Scientific Literacy: Identifying and Evaluating Their programmatic Elements", in M. Matthews (ed.) International Handbook of Research in History, Philosophy and Science Teaching. Dordrecht: Springer.

Novak J. D., Gowin D. R. (1984) Learning how to learn. Cambridge: Cambridge University Press. 
Nubiola J. (1999) El taller de la filosofía. Una introducción a la escritura filosófica. Eunsa, Pamplona.

Ochoa E., et al. (2007) El ensayo: herramienta pedagógica de trabajo del estudiante. Morelia, México: UMSNH. En línea: http://dieumsnh.qfb.umich.mx/ciees2009/pdfSINED $\% 20$ concentrado/ENSAYO2009.pdf

Olivé L. (2008) Representaciones, producción de conocimiento y normatividad: un enfoque naturalizado, en Esteban y Martínez (comp.), Normas y prácticas en la ciencia. México: UNAM, Instituto de Investigaciones Filosóficas.

Paul R., Elder L. (2003) Lectura crítica. Fundación para el pensamiento crítico. En línea: http://eduteka.icesi.edu.co/pdfdir/LecturaCritica.pdf

Polanyi M. (1966) The Tacit Dimensión. Nueva York: Doubleday Garden.

Psillos D. y Niedderer H. (eds.) (2002) Teaching and Learning in the Science Laboratory. Dordrecht: Kluwer Academic Publishers.

Pryor J. Guidelines on writing a Philosophy paper. En línea: http://www.jimpryor.net/teaching/guidelines/writing.html

Rivard L. P., and Straw, S. B. (2000) The Effect of Talk and Writing on Learning Science, An Exploratory Study. Science Education, 84, 566-593.

Schatzki T.R. (1996) Social Practices. A Wittgensteinian Approach to Human Activity and the Social. Cambridge: Cambridge University Press.

Schulz R.M. (2014) Philosophy of Education and Science Education: A Vital but Underdeveloped Relationship in M. Matthews. (ed), International Handbook of Research in History, Philosophy and Science Teaching. Dordrecht: Springer.

Schwab J. J. (1962) The teaching of science as inquiry. Harvard: Harvard University Press.

Seok P., Jin S. (2011) What teachers of science needs to know about models. An overview, International Journal of Science Education, 33, 1109-1130.

Tolvanen S., Jansson J., Vesterinen V., Aksela M. (2014) How to use historical approach to teach nature of Science in chemistry education? Science \& Education, 23, 1605-1636.

Trowbridge J. E., Wandersee J. H. (1998) Theory-driven graphic organizers in J. J. Mintzes, J. H. Wandersee, \& J. D. Novak (Eds.), Teaching science for understanding. San Diego: Academic Press.

Turner S. (1994) The Social Theory of Practices. Chicago: The University of Chicago Press.

Van Berkel B., De Vos W., Verdonk A.H., Pilot A. (2000) Normal Science Education and its Dangers: The case of School Chemistry. Science \& Education 9, 125-159.

Van Aalsvort J. (2004) Logical positivism as a tool to analize the problema of chemistry's lack of relevance in secondary school chemical education. International Journal of Science Education, 26, 1151-1168.

Wandersee J. H., and Baudoin Griffard P. (2002) The history of chemistry: Potential and actual contributions to chemical education, in J. K. Gilbert, et al. (Eds.), Chemical education: Towards research-based practice. Dordrecht: Kluwer.

Wandersee J. H. (1990) Concept mapping and the cartography of cognition. Journal of Research in Science Teaching, 27, 923-936.

Watts M. (1991) The science of problem-solving. A practical guide for science teachers. London: Cassell. 
Anexo I. Ejemplos de diagramas heurísticos

TITULO Los rayos catódicos (RC) a la espera de un observador paradigmático.

HECHOS 1803, Dalton plantea su teoría atómica, según la cual los átomos son las partículas más pequeñas de materia (1). 1833,

surgen los primeros indicios de la estructura eléctrica de los átomos gracias a las investigaciones de Faraday en electrólisis (1).

1855, Plücker produce RC motivado por la investigación de la conductividad eléctrica de gases a baja presión (1). 1890, Arthur Schuster determina el valor $\mathrm{m} / \mathrm{e}$ de los RC (2). 1897, J. J. Thomson calcula el valor $\mathrm{m} / \mathrm{e}$, sus resultados lo conducen a publicar que los RC son "corpúsculos" que constituven todos los elementos (3). Ha sido reconocido como el descubridor del electrón (4). PREGUNTA De acuerdo con la publicación de sus investigaciones ¿qué aspectos metodológicos impidieron que Schuster, habiendo calculado el valor $\mathrm{m} / \mathrm{e}$ antes que Thomson, lograra concluir que los RC estaban constituídos por partículas subatómicas?

\begin{tabular}{|l|}
\hline \multicolumn{1}{|c|}{ CONCEPTOS } \\
\hline APLICACIONES \\
Estudio de la conductividad eléctrica de gases a \\
baja presión, de la aplicación de la ley de \\
Faraday en gases y de la naturaleza misma de \\
los RC (1) (3) (5). Cinescopio, osciloscopio. \\
\hline LENGUAJE
\end{tabular}

\section{LENGUAJE}

Átomo, rayos catódicos, carga eléctrica,

materia, elementos, gases, baja presión, proporción m/e, electrón, electrólisis, corpúsculos, equivalente electroquímico, cátodo, partícula subatómica, ley de Faraday, ion hidrógeno, masa.

\begin{tabular}{|c|c|c|}
\hline \multicolumn{3}{|c|}{$\begin{array}{l}\text { PROCEDIMIENTO PARA LA OBTENCIÓN DE DATOS } \\
\text { Revisión y comparación de objetivos, resultados e inter } \\
\text { las publicaciones de A. Schuster (5) (6) y de J. J. Thomso } \\
\text { artículo que analiza y compara sus investigaciones (2) y } \\
\text { cuestiona si realmente fue Thomson el descubridor del } \\
\text { PROCESAMIENTO DE DATOS PARA OBTENER UN RESU }\end{array}$} \\
\hline & Objetivo & $\begin{array}{l}\text { Resultados } \\
\text { valor } \mathrm{m} / \mathrm{e}\end{array}$ \\
\hline $\begin{array}{l}\text { Schuster } \\
(1890)\end{array}$ & $\begin{array}{l}\text { Verificar la ley } \\
\text { electrolítica de } \\
\text { Faraday en el caso } \\
\text { de gases (2) (5) (6) }\end{array}$ & $\begin{array}{l}\quad 10^{-5}-10^{-3} \\
\text { El valor } \mathrm{m} / \mathrm{e}^{-} \mathrm{H}^{+} \\
\left(10^{-4}\right) \text { cae dentro } \\
\text { de estos límites (5) }\end{array}$ \\
\hline $\begin{array}{c}\text { Thomson } \\
\text { (1897) }\end{array}$ & $\begin{array}{l}\text { Obtener más } \\
\text { información sobre } \\
\text { la naturaleza de } \\
\text { los RC (3) (2) }\end{array}$ & $\begin{array}{l}\quad 10^{-7} \\
\text { Por } 2 \text { métodos, in- } \\
\text { dependientemente } \\
\text { del gas en que se } \\
\text { conducen (3) (2) }\end{array}$ \\
\hline
\end{tabular}
METODOLOGIA

\section{MODELO}

N. R. Hanson. La visión es una acción que lleva una "carga teórica".

La física no es solamente un sistema de exposición de los sentidos al mundo; también es una manera de pensar acerca del mundo, una manera de formar concepciones.

El observador paradigmático no es el hombre que ve y comunica lo que todos los

observadores normales ven y comunican, sino el hombre que ve en objetos familiares lo que nadie ha visto anteriormente (7). RESPUESTA Schuster no se acercó a concluir qunensamente más pequeños en masa que los átomos.

ir que los RC estaban constituidos por una partícula subatómica, debido a que el objetivo de su experimentación era verificar su teoría de que también en gases se satisfacía la ley de Faraday, no consideró los RC en su metodología (2) (5) (6). De acuerdo con el modelo de Hanson, sus observaciones no se limitaban a observar el fenómeno, sino a que fueran coherentes con un conocimiento establecido, sus observaciones estaban sin duda, "cargadas de teoría". Vio lo que un observador normal ve (7), "...la existencia separada de un átomo de electricidad nunca se me ocurrió posible, ... difícilmente hubiera sido considerado un físico serio..." (6). J. J. Thomson, un observador paradigmático, estuvo dispuesto a olvidar todo lo que conocía y contemplar los sucesos como si fuera un niño (7). Así, "...sus experimentos lo guiaron a concluir, proponer y defender una atrevida idea que revolucionó la física: que el átomo no era atómico..." (4).

REFERENCIAS (1) Mahan B. (1977) Química. Fondo Educativo Interamericano, pp. 397-401. (2) Feffer S. (1989) Arthur Schuster, J. J. Thomson, and the discovery of the electron. Historical Studies in the Phisical and Biological Sciences. 20: 33-61. (3) Thomson J. J. (1897). Cathode Rays. Philosophical Magazine 44: $293-316$. (4) Achinstein, P. (2001). Who really discovered the electron? En Buchwald, J.Z. \& Warwick, A. Histories of the electron. The birth of microphysics. Cambridge: The MIT Press. pp. 403-424. (5) Schuster A. (1890). The discharge of electricity trough gases. Procedings of the Royal Society. 47: 527-559. (6) Schuster A. (1911). The progress of physics. Cambridge University Press. (7) Hanson N.R. (1958) Observación. En Olivé L., Pérez Ransanz A. R. (1989). Filosofía de la ciencia: teoría y observación. S. XXI, IIF, pp. 216-252 


\begin{tabular}{|c|c|c|}
\hline \multicolumn{2}{|c|}{ El desarrollo instrum entalde la oceanografía quím ica en el contexto de las tradicionesexperimentales: el caso de la sonda CTD } & Pts. \\
\hline \multicolumn{2}{|c|}{$\begin{array}{l}\text { H ECH OS } \\
\text { En } 1958 \text {, Niel Brown y Bruce Hamon -de la Division of Fisheries and Oceanography of the Commonwealth Scientific and Industrial Research } \\
\text { Organization of Australia- publicaron en el burnal of Scientific Instruments un artículo titulado «A temperature-chlorinity-depth recorder } \\
\text { for use at sea». En él se describe un nuevo instrumento para registrar la temperatura, clorinidad y profundidad en los primeros mil metros } \\
\text { del mar; éste resuelve tres problemas de los métodos estándar usados hasta ese entonces: la discontinuidad de los datos registrados, el } \\
\text { límite operacional de } 400 m \text { de profundidad, y la optimización del soporte físico y suministro de energía. La sonda STD (salinity, temperature } \\
\text { and depth por sus siglas en inglés), posteriormente llamada CTD (Conductivity en vez de salinity), representa un fulcro importante en la } \\
\text { oceanografía moderna pues no sólo se ha convertido en un artefacto de uso primordial en los estudios oceanográficos sino que en su } \\
\text { momento ayudó a robustecer, dentro de la comunidad científica interesada en los estudios del océano, las mediciones de conductividad } \\
\text { para la salinidad, que a su vez ayudó al consenso de una nueva escala de salinidades prácticas, avalada por la UNESCO en } 1980 .\end{array}$} & 3 \\
\hline \multicolumn{2}{|c|}{$\begin{array}{l}\text { PREG U N TA } \\
\text { ¿Cómo fue a grandes rasgos el proceso de construcción y estabilización del fenómeno de la temperatura y salinidad en el océano -en otras } \\
\text { palabras, cuál fue el contexto histórico de las estructuras heurísticas de inferencias y adecuación técnica (Martínez, 2003) en esta área de la } \\
\text { oceanografía-cristalizado en el diseño y el uso extendido de la sonda CTD desarrollada por Brown y Hamon? }\end{array}$} & 3 \\
\hline \multirow{3}{*}{$\begin{array}{l}\text { CONCEPTOS } \\
\text { Aplicaciones } \\
\text { La sonda CTD es utilizada prácticamente en } \\
\text { cualquier campaña oceanográfica actual como } \\
\text { parte del protocolo básico de generación de un } \\
\text { perfil oceanográfico, sea en la recolección de datos } \\
\text { o en el estudio de un proceso o fenómeno. }\end{array}$} & METODOLOGIA & \\
\hline & $\begin{array}{l}\text { Proce dim ie n to para o b te n ción de d a tos } \\
\text { Revisión de documentos históricos } \\
\text {-Artículo de Brown y Hamon (1958) } \\
\text {-Documentos técnicos de la UNESCO sobre ciencias del mar (1984) }\end{array}$ & 1 \\
\hline & $\begin{array}{l}\text { Revisión de documentos historiográficos } \\
\text { - Baker, Ocean Instruments and Experiment Design (1980) } \\
\text { Entrevista } \\
\text {-Dr. Artemio Gallegos (Instituto de Ciencias del Mar y Limnología, UNAM) }\end{array}$ & 1 \\
\hline $\begin{array}{lr}\text { Le n g u a je } & \\
\text {-Oceanografía química } & \text {-Tradición experimental } \\
\text {-Salinidad } & \text {-Estructura heurística }\end{array}$ & $\begin{array}{l}\text { Pro ce sa m ie n to de lo s da to s p a ra o b te n e r u n re su lta do } \\
\text {-Lectura crítico analítica de los documentos históricos e historiográficos. } \\
\text {-Diagramas conceptuales. }\end{array}$ & 1 \\
\hline $\begin{array}{ll}\text {-Temperatura } & \text {-Prácticas científicas } \\
\text {-Conductividad } & \\
\end{array}$ & -Entrevista a investigador de la unidad académica "Procesos oceánicos y costeros". & 1 \\
\hline $\begin{array}{l}\text { M o d e lo } \\
\text { Modelo evolutivo de los métodos experimentales } \\
\text { en la ciencia y la autonomía de las tradiciones } \\
\text { experimentales (Martínez, 2003) }\end{array}$ & $\begin{array}{l}\text { A n álisis/ co n clu sión de rivada de los da tos } \\
\text {-Las propuestas de optimización técnica en oceanografía parten de una compleja y } \\
\text { vasta estructura heurística previa (Brown y Hamon retomaron las bases sobre un } \\
\text { sistema de registro continuo de la conductividad y la temperatura hecho por } \\
\text { Jacobsen en 1948). } \\
\text {-La práctica científica dentro de una tradición como la oceanografía, prejuzga las } \\
\text { aserciones y técnicas procedimentales que habrán de dotar de sentido y } \\
\text { funcionalidad a sus propios recursos epistémicos, permitiendo el progreso metódico } \\
\text { dentro de un marco no problematizado desde otros criterios (el horizonte de mejoras } \\
\text { posibles del CTD estaba ya delineado por la maniobrabilidad de los aparatos así como } \\
\text { a la implementación de resistores variables). } \\
\text {-El uso exhaustivo de los instrumentos científicos y la comparación entre resultados } \\
\text { por distintos grupos de investigación oceanográfica conectados institucionalmente, } \\
\text { habilita el tomar conciencia de la falibilidad de las evidencias iniciales de medición y } \\
\text { modelaje, pero sólo cuando surgen contradicciones y fracasos en las prácticas } \\
\text { habituales (la modificación propuesta de la escala de salinidades prácticas, por la } \\
\text { UNESCO, es muestra de ello; el uso del CTD fue parte importante). }\end{array}$ & 2 \\
\hline \multicolumn{2}{|c|}{$\begin{array}{l}\text { RESPU ESTA / R ESU LTA D O } \\
\text { La aportación teórico-instrumental de Brown y Hamon se erige sobre tres pilares de la tradición de las ciencias del mar: 1) la continuidad (y } \\
\text { los datos disponibles) de las expediciones marítimas desde mediados del siglo XIX, 2) la configuración del problema de la circulación } \\
\text { oceánica -y con él las mediciones de temperatura y salinidad-como fin epistémico prioritario a partir de } 1960 \text { y, 3) la estrecha relación } \\
\text { entre práctica científica oceanográfica y el uso de tecnología (sustentada en ingeniería y teoría físico-matemática) desde la configuración } \\
\text { (tanto conceptual como institucional-industrial) de la subdisciplina de la oceanografía química a partir de 1930. }\end{array}$} & 3 \\
\hline \multirow{2}{*}{\multicolumn{2}{|c|}{$\begin{array}{l}\text { REFER EN CIA S } \\
\text { De los hechos: } \\
\text {-Hamon, B., Brown, N., Journal Of Scientific Instruments (1923-1967) Volume: } 35 \text { Issue: } 12 \text { (1958-12-01) p. 452-458. ISSN: 0950-7671 } \\
\text {-UNESCO. Documentos técnicos de la Unesco sobre ciencias del mar (1984). «10 informe del Grupo Mixto de Expertos sobre Tablas y } \\
\text { Patrones Oceanográficos», París, Francia. } \\
\text {-Baker, J. (1980), «Ocean Instruments and Experiment Design» en Bruce Warren, and Carl Wunsch, (1980), Evolution of Physical } \\
\text { Oceanography, Spring 2007. Massachusetts Institute of Technology. } \\
\text { De los conceptos: } \\
\text {-Paytan, A. (2006), Marine Chemistry. Lecture } 1 \text { - Introduction: Chemical Oceanography the Big Picture. Standford University, Spring } 2006 . \\
\text { De la metodología: } \\
\text { Martínez, S., (2003), Geografía de lasprácticas científicas, UNAM-Instituto de Investigaciones Filosóficas, México, D.F. } \\
\text { A U TO EV A LU A CIO N }\end{array}$}} & 3 \\
\hline & & \\
\hline
\end{tabular}


Anexo II. Temas y lecturas que conforman el plan de estudios

Introducción al conocimiento científico

- Bachelard, G. (1979). La noción del obstáculo epistemológico en La formación de espiritu cientifico, México, Siglo XXI.

- Hacking, I. (1996). Lo real y las representaciones, Microscopios, en Representar e Intervenir, México: Paidós-UNAM.

- Kneller, G. (2009). De las conjeturas a los paradigmas y de los programas de investigación a los anteproyectos metafísicos en Chamizo J.A., (compilador) Aspectos filosóficos y sociales de las ciencias, México: UNAM.

Construcción social del conocimiento científico

- Hacking, I. (1999). Rocas en ¿La construcción social de quée, Paidós, Barcelona.

- Knorr Cetina, K. (1983). La fabricación del conocimiento. Buenos Aires: Editorial Universidad Nacional de Quilmes.

- Martínez S. (2005). La geografía de la razón científica: dependencia epistémica y estructura social de la cognición, en Martínez, S.F. y Godfrey G. Historia, filosofía y enseñanza de la ciencia. México: IIFUNAM.

- Richards, S. (2009). Estudios Sociales de ciencia y tecnología en Chamizo J.A., (compilador) Aspectos filosóficos y sociales de las ciencias, México: UNAM.

Tecno-química

- Bensaude-Vincent B., Loeve S., Nordman A., Schwartz A. (2011) Matters of Interest: The Objects of Research in Science and Technoscience, Journal of General Philosophy of Science, 42:365-383.

- Chamizo, J. A. (2013). Technochemistry: One of the chemists' ways of knowing. Foundations of Chemistry 15:157 170 .

- Sismondo S. (2009). Actor-Network Theory en An Introduction to Science and Technology Studies, New York: Wiley.

\section{Lenguaje}

- Foucault, M. (2005). La Prosa del Mundo en Las palabras y las cosas, Siglo XXI, México.

- Jacob, C. (2007). Análisis y síntesis. Operaciones Interdependientes entre la práctica y el lenguaje químico en Chamizo J.A., (ed.) La Esencia de la Quimica. Reflexiones sobre filosofía y educación, México: UNAM.

- Lavoisier, A. (1797). Discurso preliminar, en Tratado elemental de chímica (pp.I-XVI). México: Real Seminario de Minería de México.

- Weisberg, M. (2011). El agua no es $\mathrm{H}_{2} \mathrm{O}$ en Baird, D., Scerri, E., y McIntyre, L. (coord.)., Filosofía de la química. Sintesis de una nueva disciplina. México: Fondo de Cultura Económica.

Autonomía

- Bachelard, G. (1978). El no sustancialismo. Los pródromos de una química no-lavoiseriana en La filosofía del no. Buenos Aires: Amorrortu editores.

- Bachelard, G. (2007). La paradoja del materialismo de los filósofos. De la generalidad a la especificidad. De la homogeneidad a la pureza, en Chamizo, J. A. (comp), La esencia de la química. Reflexiones sobre filosofía y educación. México: UNAM.

- Bensaude-Vincent, B. (1998). Atomism and Positivism: A Legend about French Chemistry, Annals of Science. 56:81-94.

- Lombardi, O. y Pérez, A. R. (2010). En defensa de la autonomía de la química frente a la física. Discusión de un problema filosófico, en Chamizo, J. A (ed). Historia y filosofía de la química. Aportes para la enseñanza. México: UNAM-Siglo XXI. 
Ética

- Hoffmann R. (2015). Tension in Chemistry and Its Contents, Accountability and Research, 22, 330-345.

- Ibarra, A. y Olivé León (eds.) (2003). Cuestiones éticas de la ciencia y la tecnología en el siglo XXI, Madrid: Biblioteca Nueva.

- Kovac, Jeffrey (2011). La ética profesional en la ciencia en Baird, D., Scerri, E., y McIntyre, L. (coord.)., Filosofía de la química. Síntesis de una nueva disciplina. México: Fondo de Cultura Económica. 